\title{
Theory of Hyperspherical Sturmians for Three-Body Reactions ${ }^{\dagger}$
}

\author{
G. Gasaneo* \\ Departamento de Física, Universidad Nacional del Sur and Consejo Nacional de Investigaciones Científicas y \\ Técnicas, 8000 Bahía Blanca, Buenos Aires, Argentina
}

\section{M. Mitnik}

Instituto de Astronomía y Física del Espacio, Consejo Nacional de Investigaciones Científicas y Técnicas and Departamento de Física, Facultad de Ciencias Exactas y Naturales, Universidad de Buenos Aires. C.C. 67, Suc. 28, (C1428EGA) Buenos Aires, Argentina

\author{
A. L. Frapiccini, F. D. Colavecchia, and J. M. Randazzo \\ División Colisiones Atómicas, Centro Atómico Bariloche and Consejo Nacional de Investigaciones Científicas y \\ Técnicas, 8400 S. C. de Bariloche, Río Negro, Argentina
}

Received: May 2, 2009; Revised Manuscript Received: August 12, 2009

In this paper we present a theory to describe three-body reactions. Fragmentation processes are studied by means of the Schrödinger equation in hyperspherical coordinates. The three-body wave function is written as a sum of two terms. The first one defines the initial channel of the collision while the second one describes the scattered wave, which contains all the information about the collision process. The dynamics is ruled by an nonhomogeneous equation with a driven term related to the initial channel and to the three-body interactions. A basis set of functions with outgoing behavior at large values of hyperradius is introduced as products of angular and radial hyperspherical Sturmian functions. The scattered wave is expanded on this basis and the nonhomogeneous equation is transformed into an algebraic problem that can be solved by standard matrix methods. To be able to deal with general systems, discretization schemes are proposed to solve the angular and radial Sturmian equations. This procedure allows these discrete functions to be connected with the hyperquatization algorithm. Finally, the fragmentation transition amplitude is derived from the asymptotic limit of the scattered wave function.

\section{Introduction}

The understanding of the dynamics of the three-body problem is of fundamental importance for the atomic physics and chemistry communities. Among all the possibilities, focus on this topic recently shifted toward fragmentation or recombination processes because they appear in many physical and chemical reactions. ${ }^{1,2}$ In this report we address the theoretical treatment of fragmentation and present a method to deal with these problems.

A variety of ab initio time-independent methods have been developed to study fragmentation problems along time. Some of them have proven to be quite successful, such as the $R$-matrix, the $J$-matrix, the Close-Coupling (including the Convergent Close-Coupling), and the Exterior Complex Scaling (ECS) ${ }^{3-7}$ Even when some of these methods share some points with each other, they differ substantially in the way the boundary conditions are considered. The formulation of the ECS method is specially interesting because (i) it concentrates efforts on finding the wave function corresponding to the reaction process and (ii) it places the attention over the asymptotic region where all the particles are far from each other. In this region only fragmentation channels contribute to the wave function, and the asymptotes are represented by outgoing spherical waves. There, the ECS rotates the coordinates to the complex plane producing

\footnotetext{
† Part of the "Vincenzo Aquilanti Festschrift".

* Corresponding author, ggasaneo@criba.edu.ar.
}

an exponential decaying wave function. The strength of the exponential decay depends on the rotation angle, and for larger angles stronger decay occurs. This fact is used to numerically impose the boundary conditions; the wave function is set equal to zero on a fixed radius on each coordinate. This is justified for those regions where the exponentially decaying function can be numerically considered zero.

Very recently our group ${ }^{8-12}$ introduced a spectral method that shares some features with the ECS, in the sense that the boundary conditions are imposed in the reaction region, where all the particles are far from each other. Instead of introducing complex rotation of the coordinates, we used Sturmian functions (SF) with outgoing boundary conditions. These SF are solutions of two-particle Schrödinger equations where the magnitude of the potential is the eigenvalue. The outgoing boundary conditions enforce the SF and the eigenvalues to be complex. The solutions of the Schrödinger equation with a complex potential have necessarily outgoing or incoming flux depending on the sign of the imaginary part of the potential. Thus, the SF have all different flux depending on the eigenvalue considered. The flux can be associated to arbitrary short or long-range potential as explained in refs $9-12$. This is a systematic and physically founded way to generate absorbing or emitting optical-like potentials ${ }^{13-16}$ which re-create the flux at the boundaries. The Sturmian wave equation to be solved can be defined to be as similar as possible to the real problem under study. ${ }^{11,12,17}$ The three-body Schrödinger equation is then transformed into a 
nonhomogeneous problem where the inhomogeneity is given by the action of $(H-E)$ on the initial channel wave function, where $H$ is the three-body Hamiltonian and $E$ the energy of the system. A configuration interaction scheme can be implemented for the scattered wave function as a linear combination of products of two-particle Sturmian functions. This allows the Schrödinger equation to be transformed into an algebraic problem that can be solved by standard matrix techniques. Our proposal can be considered as a variant of the discrete variable representation of the ECS method. Two fundamental issues distinguish our methodology from the ECS: (i) we do not rotate the coordinates and, more importantly, (ii) the basis set used is founded on physical grounds. This produces a very substantial reduction of the amount of computational resources needed to solve a given problem. ${ }^{9,11,12}$

The method described before has been implemented for systems having two light and one heavy particle. ${ }^{11,12}$ In this report we extend it to general systems, by using mass weighted hyperspherical coordinates suitable for a variety of systems with different masses and interactions. The use of hyperspherical coordinates facilitates the extraction of transition amplitudes directly from the asymptotic behavior of the wave function. The methods implemented in refs $10-12$ need to be completely reformulated. Separable functions in terms of electronic coordinates are not separable in hyperspherical ones. Thus, a different set of SF from those used in those papers is necessary. In this paper we propose one way to introduce SF which are separable in hyperspherical coordinates. Angular and radial Sturmian functions are defined. The angular ones depend on five angular coordinates while the radial depends on the hyperradius only. Different types of boundary conditions are set for the radial SF. A numerical strategy which allows the solution of almost any type of angular and radial potential is discussed. Once the basis sets are defined, we apply them to solve the three-body problem. The main aim of this report is to present the general theory of the method; the application to concrete physical problems will be soon published elsewhere.

The methodology presented here can be related to the hyperquantization algorithm of Aquilanti and collaborators. ${ }^{18}$ This method, which has been successfully applied to study different reaction problems, ${ }^{19,20}$ is based on a discrete representation of the hyperspherical harmonics. This discretization considerably simplifies the calculation because it avoids the evaluation of difficult five dimensional integrals. However, in the way the method was presented, the discretization used is only valid for hyperspherical harmonics. It would be of interest to extend it to include angular and radial potentials into the basis function to increase the convergence rates of the wave function expansions. The discretization scheme implemented here is the generalization of the method used by Aquilanti and collaborators to discretize the hyperspherical harmonics.

This paper is arranged as follows. In section 2 we present the basics of the theoretical description of the Sturmian method for the three-body problem in terms of hyperspherical coordinates. We introduce a parametric separability of the Schrödinger equation in these coordinates and derive the Sturmian functions for the hyperangular and hyper-radial variables. In section 3 we develop a numerical approach to solve the algebraic equations for the system. We also present the relationship between our method and the hyperquantization algorithm of Aquilanti. The theory is extended in section 4 to compute scattering functions and cross sections for this problem. Finally, we draw some conclusions and envision future directions of this work.

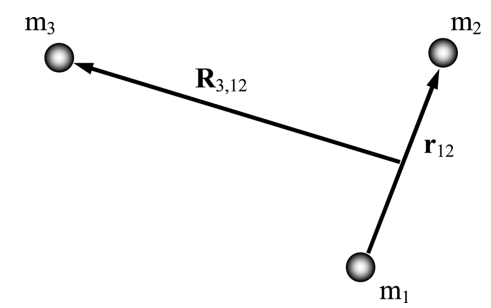

Figure 1. Jacobi coordinates for the three particles.

Atomic units are used unless otherwise noted.

\section{Generalized Hyperspherical Sturmian Theory}

2.A. Hyperspherical Coordinates. Consider three particles of masses $m_{1}, m_{2}$, and $m_{3}$. The Hamiltonian of the system can be written in terms of any of three pairs of Jacobi coordinates $\mathbf{r}_{i j}, \mathbf{R}_{k, i j}$. As usual, it is useful to use mass-scaled Jacobi coordinates $\mathbf{x}_{k}$ and $\mathbf{X}_{k}^{18}$ (see Figure 1)

$$
\mathbf{x}_{k}=\left(\frac{\mu_{i j}}{\mu}\right)^{1 / 2} \mathbf{r}_{i j} \quad \mathbf{X}_{k}=\left(\frac{\mu}{\mu_{i j}}\right)^{1 / 2} \mathbf{R}_{k, i j}
$$

for $k=1,2,3$ and $i \neq j \neq k$. The two-body reduced mass for the $i j$ pair of particles is $\mu_{i j}=m_{i} m_{j} /\left(m_{i}+m_{j}\right)$, while the threebody one is $\mu=\left(m_{i} m_{j} m_{k} /\left(m_{i}+m_{j}+m_{k}\right)\right)^{1 / 2}$. The hyper-radius $\rho$ is then defined as

$$
\rho^{2}=x_{k}^{2}+X_{k}^{2}
$$

and is independent of the particular choice of $k$. The remaining five hyperangular coordinates include the hyperangle

$$
\tan \alpha_{k}=\frac{X_{k}}{x_{k}}
$$

and the polar angles $\theta_{x_{k}}, \phi_{x_{k}}$, and $\theta_{X_{k}}, \phi_{X_{k}}$ defining the orientations $\hat{\mathbf{x}}_{k}$ and $\hat{\mathbf{X}}_{k}$ of the Jacobi vectors in the center-of-mass reference frame. We will drop the subindex $k$ in the following, so the previous definitions allow us to write $x=\rho \cos \alpha$ and $X=\rho$ $\sin \alpha$.

The kinetic energy operator takes the form

$$
T=-\frac{1}{2 \mu}\left[\frac{1}{\rho^{5}} \frac{\partial}{\partial \rho}\left(\rho^{5} \frac{\partial}{\partial \rho}\right)-\frac{\Lambda^{2}}{\rho^{2}}\right]
$$

where $\Lambda^{2}$ is the grand orbital angular momentum operator

$$
\begin{array}{r}
\Lambda^{2}=-\frac{1}{\sin ^{2} \alpha \cos ^{2} \alpha} \frac{d}{d \alpha}\left(\sin ^{2} \alpha \cos ^{2} \alpha \frac{d}{d \alpha}\right)+\frac{\mathbf{j}^{2}}{\cos ^{2} \alpha}+ \\
\frac{\mathbf{l}^{2}}{\sin ^{2} \alpha}
\end{array}
$$

where $\mathbf{j}$ and $\mathbf{l}$ denote the rotational and centrifugal angular momentum operators. Here we are using the asymmetric hyperangular parametrization. ${ }^{18}$

The Schrödinger equation for the system of particles to be considered is 
Hyperspherical Sturmians for Three-Body Reactions

$$
(T+V-E) \Psi\left(\rho, \omega_{5}\right)=0
$$

where $V$ describes the interaction potentials between the particles, and we introduced the variable $\omega_{5}$ to represent in compact form all five angular coordinates. The Sturmian functions presented here are the solutions of eq 2 but constrained by two-point boundary conditions on each coordinate. Following our recent works on atomic systems structure $^{9}$ and reaction processes, ${ }^{12}$ our first aim is to construct a suitable basis set in hyperspherical coordinates to expand the general solution of the three-body Schrödinger equation. To this end we assume the following specific forms for the interaction potentials

$$
V=\mathscr{V}(\rho, \alpha)+U(\rho)
$$

These two potentials will be in general solvable and represent a physical problem similar to the real problem to be solved. We note at this point that it would be possible to choose more general potentials depending on the internal angle $\vartheta=$ $\arccos (\hat{\mathbf{x}} \cdot \hat{\mathbf{X}})$. However, the algebra would be more cumbersome, and the introduction of this dependence at the basis level is not strictly necessary. We split each interaction potential as

$$
\begin{gathered}
\mathscr{V}(\rho, \alpha)=\mathscr{V}_{1}(\rho, \alpha)+\mathscr{T}_{2}(\rho, \alpha) \\
U(\rho)=U_{1}(\rho)+\beta U_{2}(\rho)
\end{gathered}
$$

Without loss of generality, we assume that the potential $\mathscr{T}$ includes long-range Coulomb interactions in the Jacobi-scaled coordinates given by the charges $Z_{x}$ and $Z_{X}$

$$
\begin{gathered}
\mathscr{V}_{1}(\rho, \alpha)=-\frac{Z_{x}}{x}-\frac{Z_{X}}{X}=-\frac{C(\alpha)}{\rho} \\
C(\alpha)=\frac{Z_{x}}{\cos \alpha}+\frac{Z_{X}}{\sin \alpha}
\end{gathered}
$$

while $\mathscr{T}_{2}$ is a general arbitrary, physically sound short-range potential. For example, this potential could describe the asymptotic initial state of an electron colliding with a hydrogenic system. In this case the potentials considered allow to include into the basis set the Coulomb interaction between the targetelectron and the projectile with the nucleus. We assume that $U$ depends only on the hyper-radius. The potential $U_{1}$ has a Coulomb tail $U_{1} \rightarrow-Z / \rho$ for large distances but which might not be pure Coulombic at short ones, i.e., $U_{1}=-(Z / \rho)+U_{0}(\rho)$ with $U_{0}$ a short-range potential. The potential $U_{2}(\rho)$, is a shortrange potential where its magnitude $\beta$ has been conveniently specified for our purposes. With the definitions given for the potentials and the kinetic energy the Schrödinger eq 2 is

$$
\begin{gathered}
{\left[-\frac{1}{2 \mu} \frac{1}{\rho^{5}} \frac{\partial}{\partial \rho}\left(\rho^{5} \frac{\partial}{\partial \rho}\right)+\frac{\Lambda^{2}+2 \mu \rho C(\alpha)+2 \mu \rho^{2} \mathscr{q}_{2}(\rho, \alpha)}{2 \mu \rho^{2}}+\right.} \\
U(\rho)-E] \Psi=0
\end{gathered}
$$

$$
\begin{gathered}
{\left[-\frac{1}{2 \mu} \frac{1}{\rho^{5}} \frac{\partial}{\partial \rho}\left(\rho^{5} \frac{\partial}{\partial \rho}\right)+\frac{\Lambda^{2}+2 \mu r_{\mathrm{c}} C(\alpha)+2 \mu r_{v}^{2} V_{2}\left(r_{v}, \alpha\right)}{2 \mu \rho^{2}}+\right.} \\
\left.U_{1}(\rho)+\beta U_{2}(\rho)-E\right] \Phi=0
\end{gathered}
$$

where the hyper-radius $\rho$ on the second term of (4) is replaced by two different parameters $r_{\mathrm{c}}$ and $r_{v}$ leading to potentials that do not depend on $\rho$. This enables us to define two sets of Sturmian functions, one associated to the hyper-radius $\rho$ and the other to the hyper-angle $\alpha$.

2.B. Hyperangular Sturmian Functions. The parametric separation splits the operator into an hyper-radial and angular parts, allowing eq 5 to be broken up. Hence, we can construct the following equation

$$
\begin{array}{r}
{\left[\Lambda^{2}+2 \mu r_{\mathrm{c}} C(\alpha)+2 \mu r_{v}^{2} V_{2}\left(r_{v}, \alpha\right)\right] \Omega\left(\omega_{5}\right)=} \\
\lambda(\lambda+4) \Omega\left(\omega_{5}\right)
\end{array}
$$

which is the angular Sturmian eigenvalues equation for the eigenfunctions $\Omega\left(\omega_{5}\right)$ and the eigenvalues $\lambda$. The parameters $r_{\mathrm{c}}$ and $r_{v}$ are assumed to be constants. The potentials $C(\alpha)$ and $V_{2}\left(r_{v}, \alpha\right)$ of (6) depend only on $\alpha$. Thus, a further adequate factorization for $\Omega\left(\omega_{5}\right)$ is

$$
\Omega_{v}\left(\omega_{5}\right)=\frac{H_{\lambda j l}(\alpha)}{\sin \alpha \cos \alpha} Y_{j m_{j}}(\hat{\mathbf{x}}) Y_{l m_{l}}(\hat{\mathbf{X}})
$$

where $Y_{j m_{j}}(\hat{\mathbf{x}})$ and $Y_{l m_{l}}(\hat{\mathbf{X}})$ are spherical harmonics ${ }^{22}$ and $v$ represents all the quantum numbers associated to the five coordinates. The spherical harmonics do not depend on $\alpha$, and then the substitution of (7) into (6) leads to an additional separation of the equation into a part depending only on the hyperangle $\alpha$ and a part depending on the other hyperangular coordinates

$$
\begin{aligned}
& {\left[-\frac{\mathrm{d}^{2}}{\mathrm{~d} \alpha^{2}}+\frac{j(j+1)}{\cos ^{2} \alpha}+\frac{l(l+1)}{\sin ^{2} \alpha}+2 \mu r_{\mathrm{c}} C(\alpha)+\right.} \\
& \left.2 \mu \gamma r_{v}^{2} V_{2}\left(r_{v}, \alpha\right)\right] H_{\lambda j l}(\alpha)=(\lambda+2)^{2} H_{\lambda j l}(\alpha)
\end{aligned}
$$

The function $H_{\lambda j l}(\alpha) /(\sin \alpha \cos \alpha)$ are regular at $\alpha=0$ and $\pi / 2$ when the two-point boundary conditions

$$
H_{\lambda j l}(0)=0 \quad H_{\lambda j l}\left(\frac{\pi}{2}\right)=0
$$

are satisfied. The fulfilment of these conditions enforces the discretization of the eigenvalues, which are labeled as $\lambda_{n}$. Furthermore, because eq 8 and the boundary conditions (9) are real valued, the eigenvalues $\lambda_{n}$ are real too. In general, analytic solution of eq 8 cannot be obtained due to the presence of the potentials $C(\alpha)$ and $V_{2}\left(r_{v}, \alpha\right)$. Thus, a numerical strategy should be implemented to solve (8), which we will address in the following sections. The eigenfunctions $H_{\lambda_{n} l l}$ are orthogonal

$$
\int \mathrm{d} \alpha H_{\lambda_{n} j l} H_{\lambda_{m} j l}=\delta_{\lambda_{m} \lambda_{n}}
$$


and form a complete set of functions

$$
\sum_{m=0}^{\infty} H_{\lambda_{m} j l}\left(\alpha^{\prime}\right) H_{\lambda_{m} j l}(\alpha)=\delta\left(\alpha-\alpha^{\prime}\right)
$$

The orthogonality and closure condition of $H_{\lambda j l}(\alpha)$ allow us to write the following formulas for $\Omega_{v}$

$$
\begin{gathered}
\int \mathrm{d} \omega_{5} \Omega_{a^{\prime}} \Omega_{a}=\delta_{a a^{\prime}} \\
\sum_{a} \Omega_{a}\left(\omega_{5}{ }^{\prime}\right) \Omega_{a}\left(\omega_{5}\right)=\delta\left(\omega_{5}-\omega_{5}{ }^{\prime}\right)
\end{gathered}
$$

where $\mathrm{d} \omega_{5}$ represents the five dimensional volume element and $\delta\left(\omega_{5}-\omega_{5}{ }^{\prime}\right)$ is the symbolic product of the delta functions corresponding to all the five angular coordinates.

In the particular case in which $r_{\mathrm{c}}=r_{v}=0, H_{\lambda_{n} j}$ reduces to

$$
\begin{array}{r}
\frac{H_{\lambda_{n} j}(\alpha)}{\sin \alpha \cos \alpha}=\mathcal{N}_{\lambda_{n} j l} \cos ^{j} \alpha \sin ^{l} \alpha_{2} F_{1}(-n, l+j+n+ \\
\left.2, l+\frac{3}{2}, \sin ^{2} \alpha\right)
\end{array}
$$

where ${ }_{2} F_{1}(a, b, c, z)$ is the Gauss hypergeometric function ${ }^{22}$ and $\mathcal{N}_{\lambda_{n} j l}$ is a normalization constant, and the separation constant is defined as $\lambda_{n}=j+l+2 n$, where $n$ is zero or a positive integer. The Gauss function can be related to the Jacobi polynomial. ${ }^{18}$

2.C. Hyper-Radial Sturmian Functions. The parametric separation and the eigenvalue equation defined for $\Omega_{a}\left(\omega_{5}\right)$ suggests also to factorize the full wave function $\Phi\left(\rho, \omega_{5}\right)$ as the product of the radial and the angular functions $\mathcal{S}_{\beta, \lambda}(\rho)$ and $\Omega_{a}\left(\omega_{5}\right)$, respectively

$$
\Phi\left(\rho, \omega_{5}\right)=\int_{\beta, \lambda}(\rho) \Omega_{a}\left(\omega_{5}\right)
$$

Inserting $\Phi\left(\rho, \omega_{5}\right)$ into eq 5 results in

$$
\begin{aligned}
\int_{\beta, \lambda}(\rho) \frac{\lambda(\lambda+4)}{2 \mu \rho^{2}} \Omega\left(\omega_{5}\right)+ & \Omega_{a}\left(\omega_{5}\right)\left(-\frac{1}{2 \mu} \frac{1}{\rho^{5}} \frac{\partial}{\partial \rho}\left(\rho^{5} \frac{\partial}{\partial \rho}\right)+\right. \\
& \left.U_{1}(\rho)+\beta U_{2}(\rho)-E\right) \int_{\beta, \lambda}(\rho)=0
\end{aligned}
$$

Separating the radial and the angular parts, we end up with the following equation for the hyper-radial function $\int_{\beta, \lambda}(\rho)$

$$
\begin{array}{r}
{\left[-\frac{1}{2 \mu} \frac{1}{\rho^{5}} \frac{\partial}{\partial \rho}\left(\rho^{5} \frac{\partial}{\partial \rho}\right)+\frac{\lambda(\lambda+4)}{2 \mu \rho^{2}}+U_{1}(\rho)-E\right] \int_{\beta, \lambda}(\rho)=} \\
-\beta U_{2}(\rho) \int_{\beta, \lambda}(\rho)
\end{array}
$$

This equation depends not only on the hyper-radius $\rho$ but also on the energy $E$, the angular eigenvalue $\lambda$, and $\beta$, which is the magnitude of the potential $U_{2}$. We can consider (13) as the Sturmian equation for the eigenfunctions and their eigenvalues $\beta$, while the energy $E$ (either negative or positive) is kept fixed as an external parameter. ${ }^{8-10}$ The potentials $U_{1}(\rho)$ and $U_{2}(\rho)$ are referenced as the auxiliary and the generating potential, respectively. Following, ${ }^{8,9}$ we introduce the reduced function

$$
\int_{\beta, \lambda}(\rho)=\frac{S_{\beta, \lambda}(\rho)}{\rho^{5 / 2}}
$$

into (13) leading to the following equation for $S_{\beta, \lambda}(\rho)$

$$
\left[-\frac{1}{2 \mu} \frac{\mathrm{d}^{2}}{\mathrm{~d} \rho^{2}}+\frac{\lambda(\lambda+4)-\frac{15}{4}}{2 \mu \rho^{2}}+U_{1}(\rho)-E\right]_{\beta U_{2}(\rho) S_{\beta, \lambda}}
$$

This equation has the same form as those used in our previous works, defining the two-body Sturmian problem in the radial coordinate. ${ }^{8,9}$ Boundary conditions at two points are necessary to define the Sturm-Liouville problem that defines the properties of the set of eigenvalues $\beta_{m}$. The first one at the boundary $\rho=0$ is

$$
S_{\beta, \lambda}(\rho=0)=0
$$

which ensures the regularity of $\mathcal{S}_{\beta, \lambda}(\rho)$ at the origin where divergences of the potentials might occur. The second condition determines the behavior of the solution at large distance and depends on the energy $E$. Negative energy values require an exponentially decreasing behavior at large distances. An additional logarithmic factor arises when long-range Coulomb potentials are present on the equation. Assuming, e.g., that $U_{1}(\rho)$ $=-(Z / \rho)+U_{0}(\rho)$ with $U_{0}$ a short-range potential, the condition to be satisfied by $S_{\beta, \lambda}$ at large distances is

$$
\left[\frac{\mathrm{d} S_{\beta, \lambda}(x)}{\mathrm{d} x}+\left(\mathscr{K}-\frac{Z}{\mathscr{K}(x)}\right) S_{\beta, \lambda}(x)\right]_{x=R}=0, \quad R \rightarrow \infty
$$

Here $\mathscr{K}=(2 \mu|E|)^{1 / 2}$. For positive energies there are three possible asymptotic behaviors. We can enforce $S_{\beta, \lambda}(\rho)$ to have incoming $(-)$ or outgoing $(+)$ wave boundary conditions with

$$
\left[\frac{\mathrm{d} S_{\beta, \lambda}(x)}{\mathrm{d} x} \pm i\left(K+\frac{Z \mu}{K x}\right) S_{\beta, \lambda}(x)\right]_{x=R}=0 \quad R \rightarrow \infty
$$

for long-range potentials. Here $K=(2 \mu E)^{1 / 2}$. The conditions for short-range potentials $U_{1}(\rho)$ arise simply by setting $Z=0$ in (17) or (18) for negative or positive energies, respectively. Alternatively, standing wave behavior at large distances can be imposed setting

$$
S_{\beta, \lambda}(R)=0, \quad R \rightarrow \infty
$$

The conditions (16) and (17) are real-valued, as well as the hyper-radial Sturmian equation. Thus, the spectrum decomposes in real and discrete eigenvalues. The discretization of the spectrum occurs due to the fact that the boundary condition (17) is imposed at a finite value of $\rho=R$. This is true for all the possible energetic situations considered. The standing wave boundary conditions (16) and (19) are also real as well as the 
Sturmian functions and eigenvalues. The situation is different for the incoming and outgoing waves of positive energies. The boundary conditions (18) are complex; the first-order solution of this equation is satisfied by the eikonal wave $\exp ( \pm i k x+$ $(Z \mu / K) \ln K x)$ that reduces to the usual plane wave for shortrange potentials. The combination of these functions with the factor $\rho^{-5 / 2}$ gives rise to spherical-and distorted-spherical waves boundary conditions. This type of behavior at the border leads to complex Sturmian eigenfunctions and eigenvalues of eq 15. It is well-known from the standard quantum theory, ${ }^{23}$ that the presence of an imaginary potential in the Schrödinger equation produces solutions with incoming or outgoing flux at large distances, directly associated with the magnitude of the imaginary part of the potential. Thus, this method brings a systematic and physically founded way to generate optical-like absorbing and emitting potentials ${ }^{13-16}$ to be used as an alternative to the complex dilation method. ${ }^{24}$ Moreover, our method generates a complete set of potentials allowing the representation of almost any arbitrary flux at the boundaries.

The hyper-radial eigenfunctions $S_{\beta, \lambda}$ form an orthogonal and complete set such that

$$
\int \mathrm{d} \rho S_{\beta_{n}, \lambda} U_{2}(\rho) S_{\beta_{m}, \lambda}=\delta_{\beta_{m} \beta_{n}}
$$

and

$$
\sum_{i} S_{\beta_{i}, \lambda}\left(\rho^{\prime}\right) U_{2} S_{\beta_{i}, \lambda}(\rho)=\delta\left(\rho-\rho^{\prime}\right)
$$

The full set of hyperspherical Sturmian functions satisfying eq 5 are then

$$
\Phi_{\beta_{i} \lambda_{n} v}\left(\rho, \omega_{5}\right)=\frac{S_{\beta_{i}, \lambda_{n}}(\rho)}{\rho^{5 / 2}} \frac{H_{\lambda_{j l}}(\alpha)}{\sin \alpha \cos \alpha} Y_{j m_{j}}(\hat{\mathbf{x}}) Y_{l m_{l}}(\hat{\mathbf{X}})
$$

where $v=\left\{j, m_{j}, l, m_{l}\right\}$. These functions depend parametrically on $r_{\mathrm{c}}$ and $r_{v}$ and on the charges $Z_{x}, Z_{X}$ (which define the potential $C(\alpha)$ ), and on $Z$ (related to the asymptotic Coulomb tail of $U_{1}$ ). These parameters might be used to determine the proper asymptotic behavior for atomic or molecular structure calculations $^{9}$ as well as for collisional processes. ${ }^{12}$ The basis function $\Phi_{\beta_{i} \lambda_{n} v}\left(\rho, \omega_{5}\right)$ satisfy the following orthogonality and closure relations

$$
\begin{gathered}
\int \mathrm{d} v \Phi_{\beta_{i} \lambda_{m} v^{\prime}}\left(\rho, \omega_{5}\right) U_{2}(\rho) \Phi_{\beta_{i} \lambda_{n} v}\left(\rho, \omega_{5}\right)=\delta_{\beta_{i} \beta_{i^{\prime}}} \delta_{\lambda_{n} \lambda_{n^{\prime}}} \delta_{v v^{\prime}} \\
\sum_{i, n, v} \Phi_{\beta_{i} \lambda_{n} v}\left(\rho^{\prime}, \omega_{5}{ }^{\prime}\right) U_{2}(\rho) \Phi_{\beta_{i} \lambda_{n} v}\left(\rho, \omega_{5}\right)=\delta\left(\rho-\rho^{\prime}\right) \delta\left(\omega_{5}-\omega_{5}{ }^{\prime}\right)
\end{gathered}
$$

\section{A Numerical Approach To Solve the Sturmian Equations}

3.A. Finite Differences in Uniform Lattices. The main equations that we need to solve to define the basis functions are the angular eq 8 and the radial eq 15. In general it is not possible to find analytical solutions for general potentials $V_{1}\left(r_{v}, \alpha\right), U_{1}(\rho)$ and $U_{2}(\rho)$; therefore, a numerical strategy is necessary. There are many numerical approaches that can be efficiently implemented to solve one-dimensional second-order ordinary differential equations like (8) or (15). We are going to use a simple method which is highly efficient in numerical resources and allows us to connect our Sturmians theory with the hyperquantization algorithm of Aquilanti and collaborators. ${ }^{18}$

We first consider the angular-Sturmian equation (8). Following our previous papers,,${ }^{9,10}$ we propose the discretization of the wave function $H_{\lambda_{n} j}(\alpha)$ in an uniform angular lattice $\alpha_{i}=i \Delta \alpha$ with $0 \leq \alpha_{i} \leq(\pi / 2)$ and $\Delta \alpha=\left(\pi / 2 N_{\alpha}\right)$

$$
H_{\lambda_{n} j l}\left(\alpha_{i}\right) \equiv H_{\lambda_{n} j l, i} \quad i=0,1, \ldots, N_{\alpha}
$$

In this section we will drop the subindexes $\lambda_{n} j l$ for brevity, i.e., $H_{\lambda_{n} j l, i}=H_{i}$. Within a finite-difference scheme, we can approximate the second-order derivative in eq 8 up to order $O\left(\Delta \alpha^{2}\right)$ by

$$
\frac{\mathrm{d}^{2} H_{\lambda_{n} j l}}{\mathrm{~d} \alpha^{2}}=\frac{1}{\Delta \alpha^{2}}\left[H_{i+1}-2 H_{i}+H_{i-1}\right]+O\left(\Delta \alpha^{2}\right)
$$

Defining the tridiagonal matrix $\mathbf{W}$ as

$$
\left[\mathbf{W}_{\alpha}\right]_{i, i-1}=\left[\mathbf{W}_{\alpha}\right]_{i, i+1}=-\frac{1}{\Delta \alpha^{2}}
$$

for the off-diagonal elements, and

$$
\begin{array}{r}
{\left[\mathbf{W}_{\alpha}\right]_{i i}=\frac{2}{\Delta \alpha^{2}}+\frac{j(j+1)}{\cos ^{2} \alpha_{i}}+\frac{l(l+1)}{\sin ^{2} \alpha_{i}}+2 \mu r_{\mathrm{c}} C\left(\alpha_{i}\right)+} \\
2 \mu r_{\mathrm{u}}^{2} V_{2}\left(r_{v}, \alpha_{i}\right)
\end{array}
$$

for diagonal elements, the second-order approximation version of eq 8 has the matrix representation

$$
\mathbf{W}_{\alpha} \mathbf{h}=\left(\lambda_{n}+2\right)^{2} \mathbf{h}
$$

were $\mathbf{h}$ are the solution vector with elements $H_{i}$ for $i=1, \ldots$, $N_{\alpha}$. The solutions $H_{\lambda_{n} j l}(\alpha)$ depend on the parameters $r_{\mathrm{c}}$ and $r_{v}$. The boundary conditions given by (9), satisfied by the angularSturmian functions, have to be imposed on its discrete counterpart $H_{i}$, by selecting $H_{-1}=H_{N_{\alpha+1}}=0$. In this case, the discrete spectrum is generated by the zeros of the characteristic polynomial $H_{\lambda_{N}{ }^{\alpha} j l}(\lambda)=0$ at the point $N_{\alpha}$ which are the eigenvalues $\lambda_{n}$. Equation 23 can be solved by using standard matrix diagonalization subroutines, such as Lapack package. ${ }^{25}$

The eigenfunctions of eq 23 constitute an orthogonal set with respect to both $\alpha_{i}$ and $n$

$$
\begin{aligned}
& \sum_{i}^{N_{\alpha}} H_{\lambda_{n} j l, i} H_{\lambda_{m} j l, i}=\delta_{n m} \\
& \sum_{n}^{N_{\alpha}} H_{\lambda_{n} j l, i} H_{\lambda_{n} j l, i}=\delta_{i i^{\prime}}
\end{aligned}
$$

These equations define the discrete analogues of the orthogonality and completeness relations (10) and (11). 
A similar procedure can be employed to solve the radial equation (15). In this case we propose a discretization of the Sturmian $S$ :

$$
S_{\beta, \lambda}\left(\rho_{i}\right) \equiv S_{i}, \quad i=0,1, \ldots, N_{\rho}-1
$$

in the radial lattice $\rho_{i}=i \Delta \rho$, see refs ${ }^{9}$ and..$^{10}$ The function $S_{i}$ is then defined up to a given radius $R=N_{\rho} \Delta \rho$. The radial recurrence relation obtained can be expressed in matrix form as

$$
\mathbf{W}_{\rho} \mathbf{s}=-\beta \mathbf{U}_{2} \mathbf{s}
$$

where $\mathbf{U}_{2}$ is the diagonal matrix with elements $\left[\mathbf{U}_{2}\right]_{i i}=U_{2}\left(\rho_{i}\right)$. The tridiagonal matrix $\mathbf{W}_{\rho}$ has off-diagonal elements

$$
\left[\mathbf{W}_{\rho}\right]_{i, i-1}=\left[\mathbf{W}_{\rho}\right]_{i, i+1}=-\frac{1}{2 \mu} \frac{1}{\Delta \rho^{2}}
$$

while the diagonal elements are

$$
\left[\mathbf{W}_{\rho}\right]_{i i}=\frac{1}{\mu \Delta \rho^{2}}+\frac{\lambda_{n}^{N_{\alpha}}\left(\lambda_{n}+4\right)-\frac{15}{4}}{2 \mu \rho_{i}^{2}}+U_{1}\left(\rho_{i}\right)-E
$$

The three-term recurrence relation is satisfied by the polynomial $S_{i}$ which depends on the parameters $\lambda_{n}$ and $\beta$. For states with negative energy and those with positive energy and standing wave boundary conditions, the polynomial $S_{i}$ must be zero at the $S_{-1}=S_{N_{\rho}}=0$. Even though solutions to eq 23 can be obtained with the use of standard diagonalization routines, the generalized eigenvalue eq 26 is much more difficult to solve. In this case, the eigenvalues are obtained by means of iterative algorithms, ${ }^{26}$ based on sequential orthogonal rotations of the tridiagonal matrix. This procedure concentrates the computational effort on a given number of eigenvalues in increasing order of magnitude. Calculation of the corresponding eigenvectors is performed through an inverse iteration algorithm ${ }^{27}$ or by a predictor-corrector-type method.

For positive energies and incoming or outgoing wave behavior at large values of $\rho_{i}$ we set the boundary condition (18). Numerically, the fulfilment of those conditions is done by making the replacement

$$
\left[\mathbf{W}_{\rho}\right]_{N_{\rho}, N_{\rho}} \rightarrow\left[\mathbf{W}_{\rho}\right]_{N_{\rho}, N_{\rho}}-\frac{1}{2 \mu} \frac{1}{\Delta \rho^{2}}\left[\frac{S\left(\left(N_{\rho}+1\right) \Delta \rho\right)}{S\left(N_{\rho} \Delta \rho\right)}\right]
$$

in the last element of the matrix $\mathbf{W}_{\rho} .^{10,9}$ The function $S$ is the solution of the asymptotic equation (18). The diagonalization of (26) with $\mathbf{W}_{\rho}$ replaced by (28) leads to the set of eigenvectors $\mathbf{s}$ and the complex eigenvalues $\beta_{i}$ which fulfill the desired boundary conditions. The method used here is efficient for the treatment of scattering processes even when we have to deal with non-Hermitic tridiagonal matrices like $\mathbf{W}_{\rho} \cdot{ }^{10,12}$

The radial discretization on a grid of size $N_{\rho}$ gives a set of $N_{\rho}$ vectors associated to $N_{\rho}$ eigenvalues. These vectors satisfy the following orthogonality and closure relations

$$
\begin{aligned}
& \sum_{i}^{N_{\rho}} S_{\beta_{n} \lambda_{m}, i} U_{2}\left(\rho_{i}\right) S_{\beta_{n} \lambda_{m}, i}=\delta_{\beta_{n} \beta_{n}} \\
& \sum_{n}^{N_{\rho}} S_{\beta_{n} \lambda_{m}, i^{i}} U_{2}\left(\rho_{i}\right) S_{\beta_{n} \lambda_{m}, i}=\delta_{i i^{\prime}}
\end{aligned}
$$

Note that in this case the orthogonality relation includes the weight $U_{2}\left(\rho_{i}\right)$.

3.B. The Connection with the Hyperquantization Algorithm. The connection with the hyperquantization algorithm of Aquilanti and collaborators ${ }^{18}$ can now be easily established. Let us suppose for the moment, that $r_{\mathrm{c}}$ and $r_{v}$ are set equal to zero in eq 8 . In this case, the equation has analytical solutions $H_{\lambda j l}$, given by eq 12, related to the Jacobi polynomial, as described in section 2.B. The finite-difference equation (23) (the counterpart of eq 8) is solved to obtain the eigenvectors $H_{\lambda_{n} j l, i}$ and the eigenvalues $\lambda_{n}$. In Figure 2, we show one of the vectors $H_{\lambda_{j} l, i}$ plotted against the analytic $H_{\lambda j l}$. The numerical solutions tend to the exact continuum ones in the limit of $N_{\alpha} \rightarrow \infty$. The results presented in the figure for $n=9, j=2, l=1, \lambda=j+$ $l+2 n=21$ show that the convergence toward the exact functions is surprisingly fast, even for such a big $n$ number. In the figure we present calculations performed with numerical grids having 100, 50, and 25 angular points against the exact function. As few as 25 points are enough to obtain a very satisfactory representation of the exact function in the whole range of $\alpha$. When Coulomb potentials are included in the calculations through the function $C(\alpha)$, similar behaviors are observed. In Figure 3 we plotted the same function $H_{\lambda_{n} j l, i}$ for $Z_{x}$ $=-1, Z_{X}=-1$ and for $r_{\mathrm{c}}=1$ au and $r_{\mathrm{c}}=10$ au. Different values of $r_{\mathrm{c}}$ are plotted to show that the convergence rates are not altered by the Coulomb interaction.

The connection between the differential equations satisfied by hypergeometric polynomials and the finite-difference equation satisfied by discrete variable polynomials was established in chapter II of ref 28. In general, the differential equation satisfied by a hypergeometric polynomial can be transformed into a finite-difference equation as we did above. This leads to a recurrence relation which is satisfied by a higher order polynomial which depends on the discrete variable. In ref 28 , hypergeometric type polynomials are

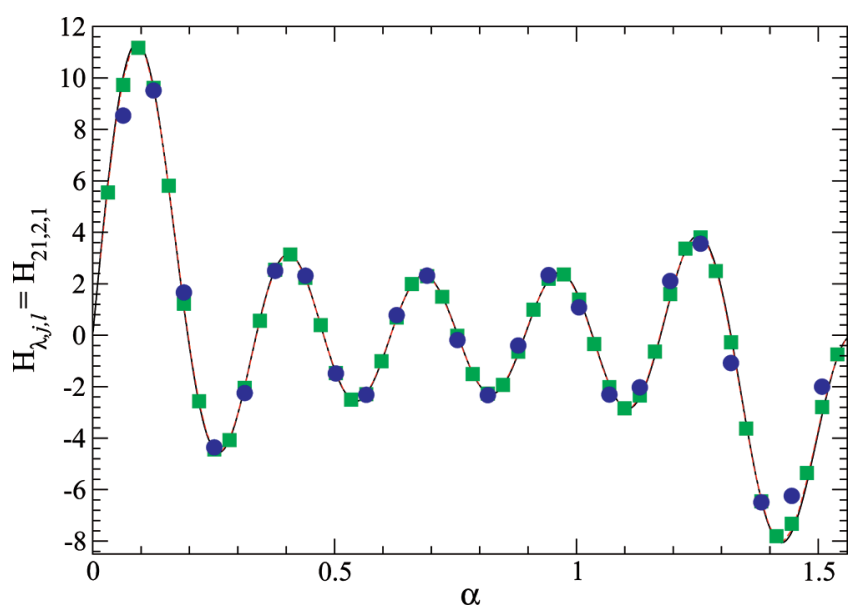

Figure 2. The vectors $H_{\lambda_{n} j l, i}$ corresponding to $r_{\mathrm{c}}=0$ and $r_{v}=0$ are plotted against $H_{\lambda j l}$ of eq 8 . The results presented are for $j=2, l=1$, $\lambda=j+l+2 n=21$. The calculations were performed with grids of 100 points, dash line, 50 points, squares, and 25 points, circles. 
discussed, but the theory explained is valid for general polynomials for which analytic expression are unknown. This is exactly what we are doing here, dealing with equations whose solutions are orthogonal polynomials that do not necessarily lead to hypergeometric functions. It is of great physical and mathematical interest to study the solutions of equations like (23), including Coulomb potentials. Aquilanti and collaborators used their expertise on the theory of orthogonal polynomials to recognize the connection we are describing. Instead of the theory of differential equation, they make use of a limiting relation for the Hahn polynomials ${ }^{18}$

$$
\begin{aligned}
\operatorname{Lim}_{X \rightarrow \infty}\left(\frac{X-2 \xi}{2}\right)^{1 / 2} Q_{n, \xi}^{\delta, \gamma, X} & = \\
& N_{n, \delta, \gamma} \cos ^{\delta} \frac{\alpha}{2} \sin ^{\gamma} \frac{\alpha}{2} P_{n}^{\delta, \gamma}(\cos \alpha)
\end{aligned}
$$

Here $Q_{n, \xi} \delta, \gamma, X$ are defined in terms of the Hahn polynomials $Q_{n}(\xi, \delta, \gamma, X)^{28}$ by the relation $Q_{n, \xi} \delta, \gamma, X=\left(w(\xi) \pi_{n}\right)^{1 / 2} Q_{n}(\xi, \delta, \gamma, X)$ and where the discretization is established through the relation

$$
\cos \alpha=\frac{X-2 \xi}{X+1}
$$

In Aquilanti's notation, $\xi$ is the discrete variable, $n$ the degree of the polynomials, and both vary in the range $0 \leq n, \xi \leq X$, with $X$ being a positive integer. The normalization constant $N_{n, \delta, \gamma}$ is given in ref 29 , while the weight function $w(\xi)$ and the norm $\pi_{n}$ of the Hahn polynomials are given in ref 30 . Aquilanti implemented the discretization of the $\cos \alpha$ into a set of $X$ points while we directly discretize the angular domain $\alpha$ directly. However, to rewrite our expressions in terms of Aquilanti's is not difficult because for each value $\alpha_{i}$ there is only one value of $\cos \alpha_{i}$ in the angular range considered. The method we are using allows the generalization of Aquilanti's procedure for general potentials included in the angular equation. This topic is part of our current investigations which we will report elsewhere.

The representation of continuous functions in terms of functions of discrete variable have also been applied to the radial coordinates. ${ }^{31,32}$ The work of Aunola related to the Coulomb problem shows that a known polynomial can be used to represent it in a discrete form the two-body Coulomb problem with angular momentum equal zero. The wave function for the $n$th bound states is represented by an orthogonal Pollaczek polynomial of order $m$. The radial coordinate is discretized on a regular grid. When the number of points of the grid tends to infinite, the discretized function tends to the exact bound state. To the best of our knowledge, no contributions have been reported for continuum states.

Aquilanti's formulation for the hyperquantization algorithm is then equivalent to ours. All the advantages of their method apply to ours; moreover, we can extend them to other situations where potentials like $V_{1}\left(r_{v}, \alpha_{i}\right)$ and/or $C\left(\alpha_{i}\right)$, are considered. In the next sections we present the application of our method to collisional problems, in which we modify our work recently presented for spherical coordinates, ${ }^{12}$ adapted to the hyperspherical ones.

\section{The Scattering Wave in Reaction Problems}

In reaction problems, the wave function is usually splitted in two terms

$$
\Psi\left(\rho, \omega_{5}\right)=\Phi_{0}\left(\rho, \omega_{5}\right)+\Psi_{\mathrm{sc}}\left(\rho, \omega_{5}\right)
$$

where $\Phi_{0}\left(\rho, \omega_{5}\right)$ is the known initial state and the scattered wave is represented by $\Psi_{\mathrm{sc}}\left(\rho, \omega_{5}\right)$. Substitution into (4) leads to the inhomogeneous Schrödinger equation satisfied by $\Psi_{\mathrm{sc}}\left(\rho, \omega_{5}\right)$

$$
(E-H) \Psi_{\mathrm{sc}}=(H-E) \Phi_{0}
$$

Typically, $\Phi_{0}$ can be written as a separable product of two known functions of the Jacobi coordinates

$$
\Phi_{0}(\mathbf{x}, \mathbf{X})=\phi(\mathbf{x}) e^{i \mathbf{K}_{0} \cdot \mathbf{X}}
$$

and satisfies the separable Schrödinger equation

$$
\left(T+V_{0}(x)-E\right) \Phi_{0}=0
$$

We assume, in the following, that the three particles are distinguishable, then the symmetrization of the wave functions is straightforward. The potential $V_{0}(x)$ is a short-range potential which supports bound states $\phi_{a}(\mathbf{x})$ with quantum numbers $a$ on the Jacobi coordinate $\mathbf{x}$. The plane wave $e^{i \mathbf{K}_{0} \cdot \mathbf{x}}$ represents an incoming particle with initial momentum $\mathbf{K}_{0}$. This initial wave
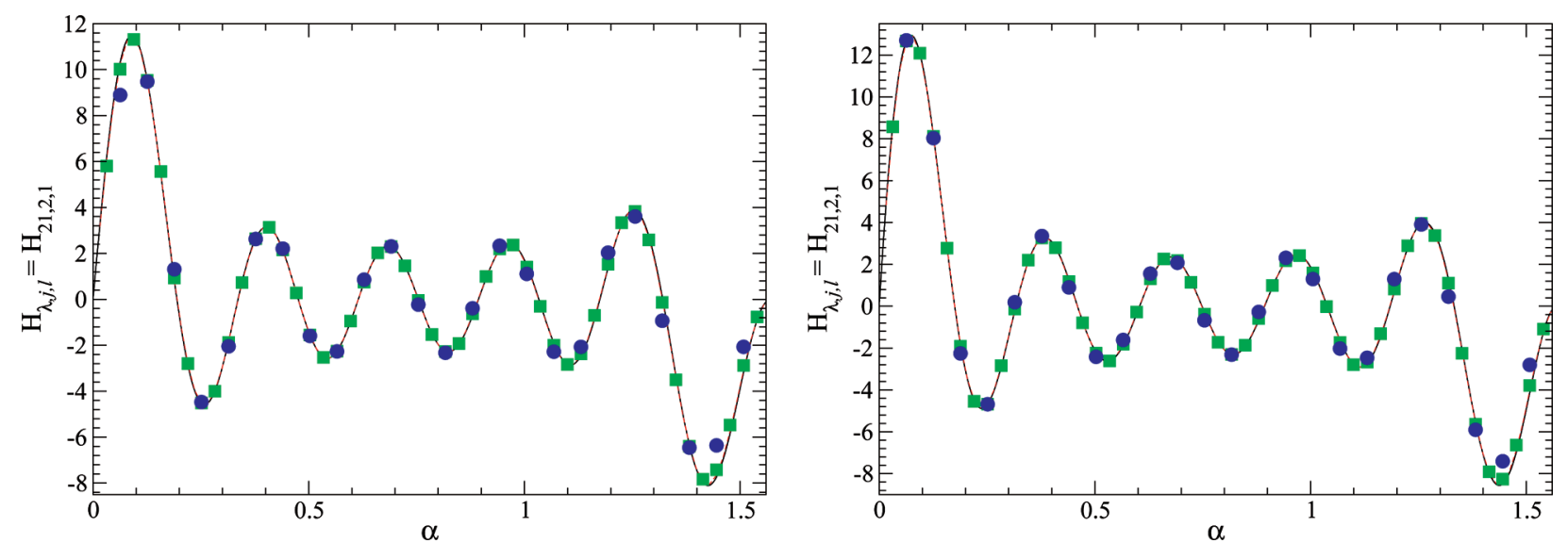

Figure 3. Convergence of numerical hyperangular functions $H_{\lambda_{n}, l}$ for $j=2, l=1, n=9$, and $\lambda_{n}=2 n+j+l=21$, including the long-range Coulomb interactions $C(\alpha)$ for different numerical grids: solid lines, 2500 points; dashed lines, 100 points; squares, 50 points; circles, 25 points. The figures show different potential parameters: left, $r_{\mathrm{c}}=1$; right, $r_{\mathrm{c}}=10$. 
function can be expressed in terms of the hyperspherical coordinates as follows

$$
\begin{aligned}
\Phi_{0}(\mathbf{x}, \mathbf{X}) & =\Phi_{0}\left(\rho, \omega_{5}\right) \\
& =\varphi_{n}(\rho \cos \alpha) Y_{j_{0} m_{j 0}}(\hat{\mathbf{x}}) \sum_{l} \sum_{m_{l}=-l}^{l} i^{l} j_{l}(\rho \sin \alpha) Y_{l m_{l}} *\left(\hat{\mathbf{K}}_{0}\right) Y_{l m_{l}}(\hat{\mathbf{X}})
\end{aligned}
$$

Here we assume that the bound state $\phi_{a}(\mathbf{x})$ has spherical symmetry, $\varphi_{n}(\rho \cos \alpha)$ is the radial part of the bound state with radial quantum number $n$, and $j_{l}(z)$ represents the spherical Bessel function of order $l .^{22}$ These are usual restrictions in the case of collisions with atomic target; however, the method can be easily extended for other reactive systems.

When the collision of the incoming particle with the bound system takes place, it produces fragmentation among other processes. The three particles evolve first under the influence of the interacting potentials, but end up as free particles leaving the reaction zone. In the case of dealing with Coulomb-like longrange interaction, the free particle waves are multiplied by distortion factors, known as the eikonal phases. Our method sets the boundary conditions to the wave function on the fragmentation region where only outgoing waves survive.

4.A. The Scattered Wave. The scattered state $\Psi_{\mathrm{sc}}$ is expanded in terms of hyperspherical Sturmians, which already account for the appropriate boundary conditions. We will restrict ourselves to the following Schrödinger equation

$$
\left[E+\frac{1}{2 \mu} \frac{1}{\rho^{5}} \frac{\partial}{\partial \rho}\left(\rho^{5} \frac{\partial}{\partial \rho}\right)-\frac{\Lambda^{2}}{2 \mu \rho^{2}}-\mathscr{T}_{2}(\rho, \alpha)-U_{2}(\rho)\right] \Psi_{\mathrm{sc}}=
$$$$
\mathscr{F}\left(\rho, \omega_{5}\right)
$$

This is a particular case of eq 4 , where the long-range interaction is suppressed; i.e., the charges $Z_{x}$ and $Z_{X}$ and the potential $U_{1}(\rho)$ were set to zero. The potentials appearing in (33) are assumed to be of short range. The function on the right-hand side $\mathscr{F}\left(\rho, \omega_{5}\right)$ represents the application of the Hamiltonian on the initial state

$$
\begin{array}{r}
\mathscr{F}\left(\rho, \omega_{5}\right)=(H-E) \Phi_{0}=\left(\mathscr{T}_{2}(\rho, \alpha)+U_{2}(\rho)-\right. \\
\left.\quad U_{0}(\rho \sin \alpha)\right) \Phi_{0}
\end{array}
$$

The scattered wave $\Psi_{\mathrm{sc}}$ is represented in terms of Sturmian basis function (7) and (14) as follows

$$
\begin{aligned}
\Psi_{\mathrm{sc}} & =\sum_{i, n, v} a_{i, n, v} \rho_{i, \lambda_{n}} \Omega_{n, v} \\
& =\sum_{i, n, v} a_{i, n, v} \frac{S_{\beta_{i}, \lambda_{n}}(\rho)}{\rho^{5 / 2}} \frac{H_{\lambda_{n} j l}(\alpha)}{\sin \alpha \cos \alpha} Y_{j m_{j}}(\hat{\mathbf{x}}) Y_{l m_{l}}(\hat{\mathbf{X}})
\end{aligned}
$$

Inserting $\Psi_{\mathrm{sc}}$ into (33) results in the equation

$$
\begin{aligned}
& \sum_{i, n, v} a_{i, n, v}\left[E+\frac{1}{2 \mu} \frac{1}{\rho^{5}} \frac{\partial}{\partial \rho}\left(\rho^{5} \frac{\partial}{\partial \rho}\right)-\right. \frac{\Lambda^{2}}{2 \mu \rho^{2}}-\mathscr{V}_{2}(\rho, \alpha)- \\
&\left.U_{2}(\rho)\right] \mathcal{S}_{i, \lambda_{n}} \Omega_{n, v}=\mathscr{F}\left(\rho, \omega_{5}\right)
\end{aligned}
$$

The action of the radial and angular differential operators on the basis functions needs to be evaluated. In a first step we use eq 8 and replace the angular momentum operator by the centrifugal barrier $\lambda_{n}\left(\lambda_{n}+4\right) / 2 \mu \rho^{2}$ and the angular potential $V_{2}\left(r_{v}, \alpha\right)$ which depends on the parameter $r_{v}$

$$
\begin{array}{r}
\sum_{i, n, v} a_{i, n, v}\left[\int_{i, \lambda_{n}}\left(-\frac{\lambda_{n}\left(\lambda_{n}+4\right)}{2 \mu \rho^{2}}+\frac{r_{v}^{2}}{\rho^{2}} V_{2}\left(r_{v}, \alpha\right)\right) \Omega_{n, v}+\right. \\
\Omega_{n, v}\left(E+\frac{1}{2 \mu} \frac{1}{\rho^{5}} \frac{\partial}{\partial \rho}\left(\rho^{5} \frac{\partial}{\partial \rho}\right)-U_{2}(\rho)\right) \mathcal{S}_{i, \lambda_{n}}- \\
\left.\mathscr{T}_{2}(\rho, \alpha) \Omega_{n, v} \mathcal{S}_{i, \lambda_{n}}\right]
\end{array}
$$

The centrifugal barrier all together with the terms of the second parentheses of the previous equation can be combined to form the left-hand side of the radial Sturmian eq 13. Defining the auxiliary potential $U_{1}$ of (13) equal to the potential $U_{2}(\rho)$ and using this equation we can rewrite (35) as follows

$$
\begin{array}{r}
\sum_{i, n, v} a_{i, n, v}\left[\beta_{i} U_{2}(\rho)+\frac{r_{v}^{2}}{\rho^{2}} V_{2}\left(r_{v}, \alpha\right)-\mathscr{V}_{2}(\rho, \alpha)\right] \mathcal{S}_{i, \lambda_{n}} \Omega_{n, v}= \\
\mathscr{F}\left(\rho, \omega_{5}\right)
\end{array}
$$

As can be seen, the Sturmian functions completely remove the kinetic energy. It is convenient to define $V_{2}\left(r_{v}, \alpha\right)$ as $\mathscr{T}_{2}(\rho, \alpha)$ with $\rho$ evaluated at $r_{v}$. In such a case, $\mathscr{T}_{2}(\rho, \alpha)$ is canceled by $\left(r_{v}{ }^{2} / \rho^{2}\right) \mathscr{T}_{2}\left(r_{v}, \alpha\right)$ around the point $\rho=r_{v}$. This point can always be selected to increase the convergence of the expansion by including in the angular basis set the relevant part of the interaction potential. Projecting eq 36 from the left onto the basis elements $\int_{i^{\prime}, \lambda_{n^{\prime}}} \Omega_{n^{\prime}, v^{\prime}}$ we obtain

$$
\begin{aligned}
& \sum_{i, n, v}\left[\beta_{i} \delta_{i i^{\prime}} \delta_{n, n^{\prime}} \delta_{v, v^{\prime}}+\left[\frac{r_{v}^{2}}{\rho^{2}}\right]_{i i^{\prime}}\left[\mathscr{T}_{2}\left(r_{v}, \alpha\right)\right]_{n, n^{\prime}} \delta_{v, v^{\prime}}-\right. \\
& \left.\left[\mathscr{V}_{2}\right]_{i^{\prime}, n^{\prime}, i, n} \delta_{v, v^{\prime}}\right] a_{i, n, v}=\mathscr{F}_{i^{\prime}, n^{\prime}, v^{\prime}}
\end{aligned}
$$

The orthogonality relation (20) was used to write the first term of this equation. $\left[r_{v}{ }^{2} / \rho^{2}\right]_{i i^{\prime}}$ and $\left[\mathscr{T}_{2}\left(r_{v}, \alpha\right)\right]_{n^{\prime}, n}$ involve only radial and angular integrations, respectively, and they are separable. $\left[\mathscr{T}_{2}\left(r_{v}, \alpha\right)\right]_{i^{\prime}, n^{\prime}, i, n}$ are the matrix elements of the interaction potential, and the integration over all the coordinates has to be performed. The function $\mathscr{F}_{i^{\prime}, n^{\prime}, v^{\prime}}$, on the right-hand side, are the projection of $\mathscr{T}\left(\rho, \omega_{5}\right)$ onto the basis functions $\int_{i^{\prime}, \lambda_{n^{\prime}}} \Omega_{n^{\prime}, v^{\prime}}$. The Sturmian functions presented in the previous sections considerably simplify the problem of solving the Schrödinger equation (33) and allow to transform it into an equivalent algebraic matrix problem $\mathbf{W a}=\mathscr{F}$. This algebraic problem can be easily solved using standard matrix techniques. The basis set diagonalizes the kinetic energy and all the interactions which depend on the hyper-radius. Thus, only the matrix elements of the interaction potential are needed. The other ingredients for the equation can be easily evaluated. For example, $\left[r_{v}^{2} / \rho^{2}\right]_{i i^{\prime}}$ requires the integration over the hyper-radius, that can be performed using Gauss-Legendre quadratures.

4.B. The Transition Amplitude. With the exact wave function for the fragmentation problem, the transition amplitude can be extracted straightforwardly. According to ref 33 the 
asymptotic limit for large $\rho$ for the scattering wave function is given by

$$
\Psi_{\mathrm{sc}} \sim-i^{1 / 2} K^{3 / 2} \frac{e^{i K \rho}}{\rho^{5 / 2}} f(\alpha, \hat{\mathbf{k}}, \hat{\mathbf{K}})
$$

The radial functions $S_{\beta_{i}}(\rho) / \rho^{5 / 2}$, eq 14 , behave at large $\rho$ as the outgoing spherical wave on (38).

Taking the asymptotic limit for $\rho \rightarrow \infty$ of $\Psi_{\text {sc }}$, eq 34 results

$$
f(\alpha, \hat{\mathbf{k}}, \hat{\mathbf{K}}) \underset{\rho \rightarrow \infty}{\approx}-\frac{i^{-1 / 2}}{K^{3 / 2}} \sum_{i, n, v} a_{i, n, v} \frac{S_{\beta_{i}}(\rho)}{e^{i K \rho}} \frac{H_{\lambda_{n} j l}(\alpha)}{\sin \alpha \cos \alpha} Y_{j m_{j}}(\hat{\mathbf{x}}) Y_{l m_{l}}(\hat{\mathbf{X}})
$$

In particular for $\rho=R$ the condition 18 is exactly satisfied and then we can obtain the following expression from the expansion for $\Psi_{\mathrm{sc}}$

$$
\Psi_{\mathrm{sc}}(\rho=R)=\frac{e^{i K R}}{R^{5 / 2}} \sum_{i, n, v} a_{i, n, v} \frac{H_{\lambda_{n} j}(\alpha)}{\sin \alpha \cos \alpha} Y_{j m_{j}}(\hat{\mathbf{x}}) Y_{l m_{l}}(\hat{\mathbf{X}})
$$

Thus, the following expression results for the transition amplitude:

$$
f(\alpha, \hat{\mathbf{k}}, \hat{\mathbf{K}})=-\frac{i^{-1 / 2}}{K^{3 / 2}} \sum_{i, n, v} a_{i, n, v} \frac{H_{\lambda_{n} j l}(\alpha)}{\sin \alpha \cos \alpha} Y_{j m_{j}}(\hat{\mathbf{x}}) Y_{l m_{l}}(\hat{\mathbf{X}})
$$

\section{Discussion and Conclusions}

In a previous paper we introduced a method to calculate three-body wave functions and transition matrices based on Sturmian functions. The basis is defined in such a way that its elements have incoming, outgoing, or standing wave behavior at large distances. These functions are solutions of Schrödinger-like wave equations where the interaction magnitudes are the eigenvalues. The potentials included in the Sturmian equations are arbitrary; however, they are chosen as the interaction potential of the physical system under study. The method presented in refs $9-12$ was based on the use of separable functions in the electronic coordinates. In this report two main goals were achieved: (a) Sturmian functions for hyperspherical coordinates with incoming, outgoing, and standing wave asymptotic conditions were developed as well as general angular-Sturmian functions depending on the hyperangle were defined and evaluated and (b) it has been shown that the method to obtain three-body wave functions and transition amplitudes can be extended to general systems.

A general discussion about how to generate the hyperangular and hyper-radial Sturmian functions was presented in section 2.B. We first showed how to define the Sturmian equation starting from the Schrödinger equation for the threebody problem under study, under a parametric separation of the wave equation. Hyperangular and hyper-radial Sturmian functions can be properly defined after this approximate separation. Different types of boundary conditions were considered for different values of the energy, which gives rise to different properties of the eigenvalue spectrum. Real eigenvalues and eigenfunctions are obtained for negative energies and also for positive energies and standing wave boundary conditions. However, for the situation where incoming or outgoing wave boundary conditions are required, complex eigenvalues and functions are found. In all the cases considered, the spectra are discrete due to the fact that the boundary conditions were imposed at finite domains of the hyper-radius. At this point it is interesting to note the differences with the standard hyperspherical approach, see, e.g., refs 35-37. In that case, first the hyperangular equation is solved assuming that the hyper-radius is a parameter. This gives rise to a set of $\rho$-dependent eigenvalues and their corresponding basis elements. In the second step, these eigenvalues are used to solve a set of coupled equations for the coefficients of the wave function for the full Schrödinger equation in that basis.

Our approach for reaction processes is based on an expansion of the scattering wave function in terms of Sturmian functions which have the correct boundary conditions at large distances. ${ }^{12}$ As was pointed out by Macek ${ }^{21}$ the use of Sturmian functions is more appropriate than adiabatic-type theories to represent continuum states.

A numerical technique to solve the angular and radial Sturmians equations was developed, using discretized versions of the Sturmians functions in uniform grids. For both the angular and the radial case a set of orthogonal discrete polynomials were obtained. These polynomials satisfy three-term recurrence relation derived from the Sturmian equation using a difference finite scheme as done in ref 28 . For general systems, the solutions are not hypergeometric polynomials. However, "freeparticle" angular Sturmians can be readly connected with the hypergeometric Hahn polynomials. This allows us to show that our method can be considered a generalization of the discretization method used by Aquilanti and collaborations. ${ }^{18}$

In section 3 we showed how to solve the three-body scattering Schrödinger equation by using the Sturmian functions. We showed that the Schrödinger equation can be transformed to an algebraic problem where only the interaction potential matrix elements are needed; see eq 37 . We also showed how the discrete Sturmian eigenfunction can be used to evaluate some of them and that they reduce to one-dimensional numeric integrals. A closed form expression for the transition amplitude is given, eq 39, by studying the asymptotic form of the scattering wave function. As we mentioned in the introduction, the main aim of this report is to present the method with detail. However, it should be mentioned that its application is straightforward. Two main collision processes are being currently studied by our group, the single and double ionization of atoms by electronimpact and radiation absorption. Reports with the results obtained will be published soon.

In summary, we have presented the theory of hyperspherical Sturmian functions for general three-body problems. Application of this method should be considered further to explore the ability of this approach to deal with the variety of different systems found nowadays in the atomic physics and chemistry literature.

Acknowledgment. The support by PGI 24/F038 of the Universidad Nacional del Sur (Argentina) and by PICT 20548/ 04 of the Agencia Nacional para la Promoción Científica y Técnica (Argentina) is gratefully acknowledged.

\section{References and Notes}

(1) Inouye, S.; Andrews, M. R.; Stenger, K.; Miesner, H. J.; StamperKurn, D. M.; Ketterle, W. Nature 1998, 392, 151.

(2) Nielsen, E.; Macek, J. H. Phys. Rev. Lett. 1999, 83, 1566.

(3) Rescigno, T. N.; Baertschy, M.; Isaacs, W. A.; McCurdy, C. W. Science 1999, 286, 2474.

(4) Bray, I.; Fursa, D. V.; Kheifets, A. S.; Stelbovics, A. T. J. Phys. B 2002, 35, R117. 
(5) Bartschat, K.; Hudson, E. T.; Scott, M. P.; Burke, P. G.; Burke, V. M. J. Phys. B 1996, 29, 115.

(6) Gorczyca, T. W.; Badnell, N. R. J. Phys. B 1997, 30, 3897.

(7) McCurdy, C. W.; Baertschy, M.; Rescigno, T. N. J. Phys. B 2004, 37, R137.

(8) Frapiccini, A. L.; Gonzalez, V. Y.; Randazzo, J. M.; Colavecchia, F. D.; Gasaneo, G. Int. J. Quantum Chem. 2007, 107, 832.

(9) Randazzo, J. M.; Frapiccini, A. L.; Colavecchia, F. D.; Gasaneo, G. Phys. Rev. A 2009, 79, 022507.

(10) Frapiccini, A. L.; Randazzo, J. M.; Gasaneo, G.; Colavecchia, F. D. Int. J. Quantum Chem. 2009, in press.

(11) Randazzo, J. M.; Frapiccini, A. L.; Colavecchia, F. D.; Gasaneo,

G. Int. J. Quantum Chem. 2009, 109, 125.

(12) Randazzo, J. M. PhD thesis, Instituto Balseiro, S. C. de Bariloche, Argentina, 2009.

(13) Seideman, T.; Miller, W. H. J. Chem. Phys. 1992, 96, 4412.

(14) Manthe, U.; Miller, W. H. J. Chem. Phys. 1993, 99, 3411.

(15) Manthe, U.; Seideman, T.; Miller, W. H. J. Chem. Phys. 1994, 101,4759

(16) Miller, W. H. J. Chem. Soc., Faraday Trans. 1997, 93, 685.

(17) Gasaneo, G.; Randazzo, J. M.; Frapiccini, A. L.; Colavecchia, F. D. Phys. Rev. A, in preparation. 3792.

(18) Aquilanti, V.; Cavalli, S.; De Fazio, D. J. Chem. Phys. 1998, 109,

(19) Aquilanti, V.; Cavalli, S.; De Fazio, D.; Volpi, A.; Aguilar, A.; Gimenez, X.; Lucas, J. M. J. Chem. Phys. 1998, 109, 721.
(20) Aquilanti, V.; Cavalli, S.; De Fazio, D.; Volpi, A. Int. J. Quantum Chem. 2001, 85, 368.

(21) Macek, J. H.; Ovchinnikov, S. Yu Phys. Rev. A 1996, 54, 544.

(22) Abramowitz, M.; Stegun, I. A. Handbook of Mathematical Functions; Dover: New York, 1972.

(23) Schiff, L. I. Quantum Mechanics; McGraw-Hill: New York, 1968.

(24) Karlsson, H. O. J. Chem. Phys. 1998, 108, 3849.

(25) Lapack Linear Algebra Package, http://www.netlib.org/lapack.

(26) Luk, F. T.; Qiao, S. Linear Algebra and Its Applications 2000, 316,171 .

(27) Hernández, V.; Román, J. E.; Tomás, A.; Vidal, V.; SLEPc Technical Report STR-2, Available at http://www.grycap.upv.es/slepc/.

(28) Nikiforov, A. F.; Suslov, S. K.; Uvarov, V. B. Classical othogonal polynomials of a discrete variable; Springer-Verlag: Berlin, 1991.

(29) Aquilanti, V.; Grossi, G.; Lagana, A. J. Chem. Phys. 1982, 76, 1587.

(30) Aquilanti, V.; Cavalli, S.; De, Fazio, D. J. Phys. Chem. 1985, 99 , 15694.

(31) Aunola, M. J. Math. Phys. 2003, 44, 1913.

(32) Aunola, M. J. Phys. A 2005, 38, 1279.

(33) Rudge, M. R. H.; Seaton, M. J. Proc. R. Soc. London, Ser. A 1965, $283,262$.

(34) Gasaneo, G.; Macek, J. H. J. Phys. B 2002, 35, 2239.

(35) Fano, U. Rep. Prog. Phys. 1983, 46, 97.

(36) Lin, C. D. Phys. Rep. 1995, 257, 1.

(37) Pack, R. T; Parker, G. A. J. Chem. Phys. 1992, 98, 6883.

JP9040869 